\title{
Rheology of Laponite-scleroglucan hydrogels
}

\author{
R. Lapasin ${ }^{\mathrm{a}, *}$, M. Abrami $^{\mathrm{b}}$, M. Grassi $^{\mathrm{a}}$, U. Šebenik ${ }^{\mathrm{c}}$ \\ a University of Trieste, Engineering and Architecture Department, Piazzale Europa, I-34127, Trieste, Italy \\ ${ }^{\mathrm{b}}$ University of Trieste, Life Sciences Department, Cattinara Hospital, Strada di Fiume 447, Trieste I-34149, Italy \\ c University of Ljubljana, Faculty of Chemistry and Chemical Technology, Vecna pot 113, 1000 Ljubljana, Slovenia
}

\section{A R T I C L E I N F O}

\section{Article history:}

Received 31 October 2016

Received in revised form 15 March 2017

Accepted 21 March 2017

Available online 23 March 2017

\section{Keywords:}

Laponite

Scleroglucan

Blending effects

Hydrogels

Nanoparticles

\begin{abstract}
A B S T R A C T
Both Laponite and scleroglucan can find several applications in various fields (from industrial to biomedical one) in virtue of their peculiar features and rheological properties displayed in aqueous phases. Structural states of Laponite dispersions strongly depend on concentration and ionic strength. When attractive and repulsive interparticle interactions are so effective that they lead to arrested states (attractive gel or repulsive glass), the rheological behavior of the dispersion undergoes a sharp transition, from quasi-Newtonian to markedly shear thinning and viscoelastic. Conversely, scleroglucan solutions gradually change to weak gels with increasing polymer concentration. The present work is concerned with aqueous Laponite-scleroglucan mixed systems, obtained according to different preparation modes, and is aimed at examining how much the content and proportion of both components affect the viscoelastic and flow properties of the mixed system.
\end{abstract}

(C) 2017 Elsevier Ltd. All rights reserved.

\section{Introduction}

Both scleroglucan, a neutral biopolymer, and Laponite, a synthetic clay, have separately found numerous applications in various industrial fields as well as in pharmaceutical and biomedical areas, by virtue of the structural features and rheological properties which are displayed, when they are dissolved or dispersed in water at sufficiently high concentration.

Scleroglucan is a nonionic polysaccharide secreted exocellularly by filamentous fungi of the genus Sclerotium. Its primary structure consists of a linear backbone of (1,3)- $\beta$-linked D-glucopyranosyl residues bearing a single $(1,6)-\beta$-linked D-glucopyranosyl unit every three sugar residues of the main chain (Rinaudo \& Vincendon, 1982). Both in aqueous solution and in the solid state, scleroglucan adopts a highly ordered, rigid, triple helical tertiary structure (triplex), which consists of three individual strands composed of six residues in the backbone per turn. The three strands of the triplex are held together by interstrand hydrogen bonds at the center of the triplex. The $(1 \rightarrow 6)$-linked $\beta$-D-glucopyranosyl side groups protrude from the outside of the triplex, so preventing intermolecular aggregation and polymer precipitation (Bluhm, Deslandes,

\footnotetext{
* Corresponding author.

E-mail addresses: rlapasin@alice.it, romano.lapasin@dia.units.it (R. Lapasin), MICHELA.ABRAMI@phd.units.it (M. Abrami), mario.grassi@dia.units.it (M. Grassi), Urska.Sebenik@fkkt.uni-lj.si (U. Šebenik).
}

Marchessault, Pérez, \& Rinaudo, 1982; Fariña, Siñeriz, Molina \& Perotti, 2001; Palleschi, Bocchinfuso, Coviello, \& Alhaique, 2005; Yanaki \& Norisuye, 1983). The triplex conformation is destabilized only in dimethyl sulfoxide or strong alkaline conditions and is characterized by a high rigidity which is responsible of the peculiar properties exhibited by aqueous scleroglucan solutions in a wide $\mathrm{pH}$ range and even at relatively high temperatures. A soft transition from sol to weak gel properties with increasing polymer concentration can be detected in both continuous and oscillatory shear tests (Grassi, Lapasin, \& Pricl, 1996; Lapasin, Pricl, \& Esposito, 1990). As triplex clustering increases leading to the formation of a threedimensional hydrogel network, shear thinning behavior becomes more marked and ultimately an apparent yield stress can be individuated from flow curves, thixotropic responses becomes more and more evident, and a progressive transition is observed in the mechanical spectra with prevailing elasticity over the whole frequency window.

Due to the marked shear thinning behavior of its hydrogels, scleroglucan is used as thickener and suspending agent to impart adequate rheological properties and improve stability of disperse systems in several industrial sectors (Lapasin \& Pricl, 1995). Nonionic polymers usually show only slight interactions with nonionic and cationic surfactants and can be conveniently employed for the preparation of stable cosmetic $\mathrm{O} / \mathrm{W}$ emulsions since both polymer and surfactant mixtures can contribute independently and positively to the stabilization of the dispersed phase (Bais, Trevisan, Lapasin, Partal, \& Gallegos, 2005). Scleroglucan is not toxic and does 
not alter blood or living tissues; when applied to skin or eyes it does not cause sensitization. In addition, it belongs to the group of biological response modifiers, which have been attributed with antitumor effects in many cases (Bohn \& BeMiller, 1995). All these characteristics are very important, especially when preparing both pharmaceutical and cosmetic emulsions.

Among biopolymers, scleroglucan and its derivatives appear to be particularly well suited for the formulation of hydrogel matrices for sustained drug release of bioactive molecules, because of their peculiar features such as high biocompatibility, biodegradability, bioadhesivity, chemical and thermal resistance and good mechanical properties (Coviello, Grassi, Lapasin, Marino, \& Alhaique, 2003; Coviello et al., 2005; Grassi, Lapasin, Pricl, \& Colombo, 1996; Grassi et al., 2009; Matricardi, Onorati, Coviello, \& Alhaique, 2006; Viñarta, François, Daraio, Figueroa, \& Fariña, 2007). In the field of enhanced oil recovery (EOR) scleroglucan is assessed as environmentally friendly viscosifying agent in virtue of its no toxicity and biodegradability. Indeed, it is quite suitable for formulating water based drilling muds to be employed in harsh environments owing to its good stability towards high salinities, temperatures and alkaline conditions, moderate interactions with surfactants and its robust shear tolerance (Baba Hamed \& Belhadri, 2009; Gallino, Guarneri, Poli, \& Xiao, 1996; Kulawardana et al., 2012; Sveistrup, van Mastrigt, Norrman, Picchioni, \& Paso, 2016).

On the other hand, Laponite is currently used as a rheology modifier in various technological applications, such as surface coatings, ceramic glazes, paints, home care and personal care products, as well as film forming agent and in pharmaceutical and nanocomposite formulations.

Laponite is a synthetic hectorite manufactured by processing combined salts of sodium, magnesium and lithium along with sodium silicate with an empirical chemical formula $\mathrm{Na}^{+0.7}\left[\left(\mathrm{Si}_{8} \mathrm{Mg}_{5.5} \mathrm{Li}_{0.3}\right) \mathrm{O}_{20}(\mathrm{OH})_{4}\right]^{-0.7}$. Laponite nanoparticles are rigid disc-shaped crystals with a thickness of $1 \mathrm{~nm}$, an average diameter of $30 \mathrm{~nm}$, a bulk density of $2.65 \cdot 10^{3} \mathrm{~kg} / \mathrm{m}^{3}$. Each platelet is composed of an octahedral magnesia sheet that is sandwiched between two tetrahedral sheets of silica. The isomorphic substitution of divalent magnesium by monovalent lithium causes a deficiency of positive charge, which is balanced by sodium atoms residing in the interlayer space. When dispersed in aqueous media the sodium ions are released, leading to a permanent negative charge distribution on both opposite faces of Laponite disks. Positive charges on the rim are due to the protonation process of the local hydroxide groups.

Because of all these peculiar platelet features, aqueous Laponite dispersions can display a variety of structural conditions and, consequently, of rheological properties for different particle concentrations and ionic strength values. Marked shear thinning or plastic behavior and highly elastic responses are exhibited when interparticle (attractive or repulsive) interactions are so effective to generate arrested states of various nature (attractive gel, Wigner or repulsive glasses). Numerous investigations have been addressed to define the state diagram of aqueous Laponite dispersions in the ionic strength vs clay concentration plane, i.e. to individuate the different regions of isotropic liquids, disordered (gels and glasses), ordered (nematic phases), flocculated states (Cummins, 2007; Gabriel, Sanchez, \& Davidson, 1996; Jabbari-Farouji, Tanaka, Wegdam, \& Bonn, 2008; Levitz, Lécolier, Mourchid, Delville, \& Lyonnard, 2000; Mourchid, Lécolier, Van Damme, \& Levitz, 1998; Mourchid, Delville, Lambard, Lécolier, \& Levitz, 1995; Mongondry, Tassin, \& Nicolai, 2005; Ruzicka \& Zaccarelli, 2011; Ruzicka, Zulian, \& Ruocco, 2004; Ruzicka, Zulian, \& Ruocco, 2006; Tanaka, Meunier, \& Bonn, 2004; Tanaka, Jabbari-Farouji, Meunier, \& Bonn, 2005). The various contradictions emerging from the comparison of the proposed Laponite dispersion state diagrams are partly apparent and can be mainly ascribed to different aging times and, secon- darily, to different protocols of samples preparation or Laponite type (Ruzicka \& Zaccarelli, 2011). Indeed, time elapsed after dispersion preparation plays a paramount role since even very low Laponite concentration dispersions can undergo aging up to a final arrested state. Regarding the different time evolution of the sol/arrested state transition in salt-free aqueous systems (ionic strength $\approx 2 \times 10^{-4} \mathrm{M}$ ), two distinct non-ergodic states (gel and repulsive glass) have been individuated at lower and higher concentrations, respectively (Ruzicka et al., 2004). In these two regions, the attractive (rim-face) and repulsive (face-face, rim-rim) electrostatic interactions between platelets are, respectively, dominant in the formation and stability of the arrested structure. At very low concentration (below $1.0 \mathrm{wt} \%$ ) the slow gelation process, originating from the attractive interparticle interactions and the relevant clustering, is followed by an extremely slow phase separation between clay-poor and clay-rich phases on the year timescale (Ruzicka et al., 2011). At even higher concentration (above $3 \mathrm{wt} \%$ ) the formation of nematic microdomains was postulated on the basis of birefringence measurements between crossed polarizers (Mourchid et al., 1998). Upon increasing ionic strength above $10^{-4} \mathrm{M}$, the role of electrostatic repulsions decreases in favor of attractive interactions between the oppositely charged edges and faces of the clay platelets. Accordingly, the amplitude of the gel region increases and the gelation time strongly decreases. At high salt concentrations, the energy barrier to particle aggregation is strongly reduced and phase separation in the form of large aggregates occurs.

Several studies have been carried out on aqueous Laponitepolymer systems, obtained by dispersing nanoparticles in polymeric matrices or adding polymer to Laponite dispersions. The scenarios drawn by the structural conditions of these mixed systems may be even more complex than those exhibited by the corresponding simple systems (water-Laponite and waterpolymer) owing to various possible interaction modes between platelets and polymer chains. The balance of different competitive mechanisms related to polymer adsorption (steric hindrance, change in superficial charge, depletion, bridging, increase in the solution viscosity) can assist or hinder the aging dynamics and the formation of a final arrested state (glass or gel), strongly depending on the polymer concentration and molecular weight. If superficially adsorbed, short flexible polymer chains can provide steric stabilization owing to excluded volume effects between polymer segments, thus inhibiting or slowing down the aggregation process when edge-to-face attractive interactions are potentially dominant. At higher molecular weights, adsorbed polymer chains may be long enough to bridge between particles, so promoting particle clustering or the formation of an associative network. Beyond particle surface saturation, depletion forces can promote particle aggregation in the presence of free nonadsorbed chains in solution. As polymer concentration increases, interactions between adsorbed layers and free chains can result in re-stabilization of clusters and, above the overlap concentration, cluster-cluster attractions increase so becoming long ranged. All these structural effects have been postulated for Laponite dispersions containing poly(ethylene oxide)(PEO) which can be considered a paradigmatic example of platelet dispersions in neutral polymer solutions, from dilute to concentrated regime (Baghdadi, Sardinha, \& Bhatia, 2005; Baghdadi, Jensen, Easwar, \& Bhatia, 2008; Kishore, Chen, Ravindra, \& Bhatia, 2015; Mongondry, Nicolai, \& Tassin, 2004; Zulian, Ruzicka, \& Ruocco, 2008; Zulian, Augusto De Melo Marques, Emilitri, Ruocco, \& Ruzicka, 2014).

As several other complex systems, Laponite-PEO systems exhibit intriguing macroscopic behavior by varying the applied shear conditions, since various structural changes can be produced on different length scales for a range of clay and PEO concentrations (Daga \& Wagner, 2006; de Bruyn, Pignon, Tsabet, \& Magnin, 
2008; De Lisi et al., 2008; Lapasin \& Maiutto, 2006; Lin-Gibson, Kim, Schmidt, Han, \& Hobbie, 2004; Maiutto, 2005; Morariu \& Bercea, 2015; Malwitz, Butler, Porcar, Angelette, \& Schmidt, 2004; Pozzo \& Walker, 2004; Schmidt, Nakatani, Butler, Karim, \& Han, 2000; Zebrowski, Prasad, Zhang, Walker, \& Weitz, 2003). Besides breakdown of particle aggregates, platelets orientation, anisotropic phase distribution or separation, which have been observed in aqueous Laponite dispersions, also a reversible 'shake gel' formation can occur over a narrow composition range, close to surface saturation. Such a shear-induced gelation can be ascribed to a polymer-clay adsorption-desorption mechanism with the formation of unstable polymer bridges.

A wide variety of nanocomposite hydrogel systems can be prepared by adding Laponite nanoparticles to chemical or physical hydrogels. The presence of nanoparticles can serve to impart unique mechanical and other physical properties to polymer hydrogels, which can lead to many diverse technological applications, mostly in biotechnological areas. Physical gels made of biopolymers, especially if unmodified, may be quite interesting in the preparation of controlled/sustained drug release systems and other biomedical devices also in virtue of their biocompatibity, nontoxicity, biodegradability characteristics. Physical hydrogels derive from weak interchain associations due to chain entanglements, hydrogen bonds, ionic bonds or strong van der Waals interactions between chains, hydrophobic interactions or crystallites bringing together two or more macromolecular chains. The added nanoparticles can be simply entrapped and distributed within the hydrogel network, as individual units or aggregates, thus acting like other solid fillers dispersed in polymeric matrices. Particle adsorption to or interactions with polymer chains can alter the network architecture, even increasing its connectivity with additional interchain physical crosslinks, thus leading to diverse extents of mutual interpenetration between the two components and heterogeneity at different length scales, up to phase separation.

As previously mentioned, aqueous Laponite dispersions display a very rich phase behavior that includes sol, gel, glass, and nematic states, depending on clay concentration, ionic strength and time of aging. Addition of ionic components generally intensifies their aging and results in a decrease in the time of transition into arrested states. The adsorption of cationic species on platelet surface induce additional attraction between them owing to the enhancement of hydrophobic interactions, thus favoring particle aggregation and reducing sedimentation stability of Laponite dispersions, as detected in the case of CTAB (cetyltrimethylammonium bromide) addition (Savenko et al., 2013). Homopolymer hydrogels made from cationic biopolymer are less frequent than those obtained from anionic polyelectrolytes such as xanthan, gellan, welan, carrageenan, alginate, pectin, carboxymethylcellulose, etc. The exact nature of the interactions between Laponite particles and anionic polymers depends subtly on the detailed structure of the polymer: type, number and separation of the anionic sites, flexibility and hydrophobicity of the backbone, branching degree and average molecular weight (Fitch, Jenness, \& Rangus, 1991). Electrostatic attractions between anionic groups and positively charged edges of Laponite particles could increase the stability of the clay dispersion on condition that the repulsive forces between platelet faces remain almost unchanged. At sufficiently high polymer concentration, when the neutralizing cations (usually sodium, potassium, calcium) effectively screen the negative charges on Laponite particles, this enhances the attractive interactions between the polymer and can result in changes in the dynamics of aging and the state of the system. The high numerical density of Laponite particles makes possible the formation of dense fractal aggregates due to the interparticle connections established by polymer chains, and eventually of a gel network structure, as observed in the case of polystyrene sulfonate addition to Laponite glass dispersion (Savenko, Bulavin, Rawiso, \& Lebovka, 2014). Rheological studies performed on Laponite/carboxy-methylcellulose blends have revealed strong synergistic effects which have been ascribed to multiple particle-chain interactions while less synergy have been observed between Laponite and xanthan gum, which has bulky trisaccharide chain (Fitch et al., 1991). The rheological responses exhibited by Laponite dispersions in aqueous xanthan matrices with added $\mathrm{NaCl}$ strongly depend on concentrations and proportion of the two components (Maiutto 2005; Lapasin, 2016). As xanthan concentration increases in dilute Laponite dispersions, the attractive particle-chain interactions give origin to synergic effects, also contributing to the connectivity of the polymer network when it is formed. As Laponite concentration increases, the contribution of the particle gel network becomes predominant over that of the added polymer. Recently, a rheological study has been performed to examine the effects of Laponite addition to alginate solutions, in particular to analyze the physical gelation due to the electrostatic interactions between the Laponite platelets and alginate chains (Davila \& d'Ávila, 2017).

Among non-ionic polysaccharides scleroglucan and its chemically analogous schizophyllan occupy a quite special position in virtue of their triple helical tertiary structure (triplex), which make possible the formation of a hydrogel network at sufficiently high polymer concentration. Aqueous concentrated Laponitescleroglucan blends appear to be worthy of consideration for biomedical applications, in particular for the preparation of hydrogel-based release systems. Laponite addition to scleroglucan weak gel could contribute to better control the drug diffusion and release kinetics as well as to improve the mechanical properties of the matrix. Starting from these practical perspectives, the present work has been addressed to study the rheological properties of aqueous Laponite-scleroglucan mixed systems, even differently prepared, sufficiently concentrated to exhibit marked viscous and elastic responses, and, in particular, to examine how much the content and proportion of both components affect the viscoelastic and flow properties of the mixed systems.

\section{Experimental}

\subsection{Materials and sample preparation}

Scleroglucano Actigum CS11 of average molecular weight $\left(\mathrm{M}_{\mathrm{W}}\right)$ $1.2 \times 10^{6}$ was provided by Cargill Inc.(USA) and used as received without further purification. Laponite ${ }^{\circledR}$ XLG (provided by Rockwood Additives Ltd, UK) is a synthetic purified hectorite with a low heavy metals content and high surface area (BET $370 \mathrm{~m}^{2} / \mathrm{g}$ ).

The most concentrated (2wt\%) Scleroglucan solution and Laponite dispersion were prepared by gradually adding the polymer powder or clay powder to distilled water under mechanical stirring. The other simple systems of lower concentration were obtained by dilution of these mother systems with distilled water under magnetic agitation.

Laponite-scleroglucan mixed systems were prepared according two different modalities: 1 ) (fresh or aged) simple systems having the same weight percent ( $2 \mathrm{wt} \%$ ) of scleroglucan or Laponite were blended in different proportions $(3: 1,1: 1,1: 3)$ under magnetic agitation; 2) Laponite was dispersed under mixing in a scleroglucan matrix ( 2 wt\%) to obtain different solid concentrations $(0.5,1$ and $2 \mathrm{wt} \%)$.

After their preparation all the systems were kept in fridge at temperature of $8^{\circ} \mathrm{C}$ for the programmed time interval before being examined. 
Table 1

$\mathrm{RBC}$ and Cross parameters of scleroglucan systems.

\begin{tabular}{|c|c|c|c|c|c|c|c|}
\hline & $C$ & (wt\%) & 0.25 & 0.50 & 1.00 & 1.50 & 2.00 \\
\hline \multirow[t]{3}{*}{$\mathrm{RBC}$} & $\eta 0$ & (Pas) & 0.25 & 18.4 & 610 & 7980 & 28500 \\
\hline & $\sigma c$ & $(\mathrm{~Pa})$ & 0.072 & 0.999 & 3.91 & 18.8 & 28.6 \\
\hline & $m$ & $(-)$ & 1.25 & 2.31 & 3.92 & 9.97 & 10.4 \\
\hline \multirow[t]{3}{*}{ Cross } & $\eta 0$ & (Pas) & 0.17 & 15.8 & 690 & 9770 & 26600 \\
\hline & $\dot{\gamma}_{c}$ & $\left(s^{-1}\right)$ & 0.48 & 0.0112 & 0.0027 & 0.0017 & 0.0011 \\
\hline & $n$ & $(-)$ & 0.59 & 0.70 & 0.81 & 0.91 & 0.92 \\
\hline
\end{tabular}

\subsection{Instrument and experimental procedures}

Experimental tests were performed at $25^{\circ} \mathrm{C}$ using the controlled stress rheometer Haake Mars III equipped with Peltier temperature control system and using the cone-plate geometry $\mathrm{C} 60 / 1^{\circ}$ (diameter $60 \mathrm{~mm}$, angle $1^{\circ}$ ) or, alternatively, the crosshatched plate-plate geometry PP35Ti (diameter $35 \mathrm{~mm}$ ). A glass solvent trap cover was used to prevent water evaporation.

By applying an increasing sequence of constant stress segments to the samples and measuring the corresponding shear rate $(\dot{\gamma})$ the steady flow behavior was determined. The stress was kept constant until the relative variation of the shear rate satisfied the following constraint, $(\Delta \dot{\gamma} / \dot{\gamma}) / \Delta \mathrm{t} \leq 0.05$, or the segment duration $(\Delta \mathrm{t})$ was no longer than the cutoff value of $100 \mathrm{~s}$. Oscillatory stress sweep tests were performed at $1 \mathrm{~Hz}$ in order to define the linear viscoelasticity range. All the frequency sweep measurements were performed within the linear range.

\subsection{Models for data correlation}

Using shear rate as independent variable, experimental viscosity data can be correlated with good approximation with the Cross equation (Cross, 1965):

$\eta=\eta_{\infty}+\frac{\eta_{0}-\eta_{\infty}}{1+(\lambda \dot{\gamma})^{n}}$

where $\eta_{0}$ and $\eta_{\infty}$ are the asymptotic values of the viscosity at zero and infinite shear rates, respectively, $\lambda$ is the characteristic time and $n$ measures the shear rate dependence of viscosity in the power law region. Alternatively, if shear stress is assumed as reference variable, a satisfactory data fitting can be provided only by the Roberts-Barnes-Carew (RBC) model, also in the case of strong shear thinning or apparently plastic behavior, when the initial gradual decrease in the viscosity is followed by its dramatic drop (Roberts, Barnes, \& Carew, 2001):

$\eta=\eta_{\infty}^{\prime}+\frac{\eta_{0}^{\prime}-\eta_{\infty}^{\prime}}{1+\left(\sigma / \sigma_{c}\right)^{m}}$

where the critical stress $\sigma_{\mathrm{c}}$ locates the transition region between the former shear thinning and the latter one while the exponent $m$ rules the rate of viscosity decrease therein. In the case of plastic behavior (high $m$ values), the apparent yield stress is given by $\sigma_{c}$. The RBC model represents a modified version of the Ellis equation (Ellis, 1927), more flexible than the original one, since the original parameters $\eta_{0}$ and $\eta_{\infty}$ are now substituted by functions $\left(\eta_{0}^{\prime}\right.$ and $\eta_{\infty}^{\prime}$ ) of the shear stress as follows:

$\eta_{0}^{\prime}=\frac{\eta_{0}}{1+\left(\sigma / \sigma_{1}\right)^{p}}$

$\eta_{\infty}^{\prime}=\eta_{\infty}\left[1+\left(\sigma / \sigma_{2}\right)^{s}\right]$

where $\sigma_{1}, \sigma_{2}, p$ and $s$ are adjustable parameters besides the asymptotic viscosity values $\eta_{0}$ and $\eta_{\infty}$.
The experimental data from oscillatory tests, namely frequency sweep, can be described quite satisfactorily with the classical generalized Maxwell (GM) model (or Maxwell-Wiechert model) composed of an elastic spring and Maxwell elements in parallel. Actually, four Maxwell elements were sufficient to describe the mechanical spectra qualitatively well.

The GM equations for describing the frequency dependence of the viscoelastic moduli are:

$G^{\prime}=G_{e}+\sum_{i=1}^{4} \frac{G_{i} \lambda_{i}^{2} \omega^{2}}{1+\lambda_{i}^{2} \omega^{2}}$

$G^{\prime \prime}=\sum_{i=1}^{4} \frac{G_{i} \lambda_{i} \omega}{1+\lambda_{i}^{2} \omega^{2}}$

where $G_{e}$ is the equilibrium modulus $(\omega \rightarrow 0)$, while $G_{i}$ and $\lambda_{\mathrm{i}}$ are the relaxation modulus and the corresponding relaxation time of the $\mathrm{i}^{\text {th }}$ Maxwell element, respectively. In order to reduce the correlation degree between the adjustable parameters, the minimization procedure was performed by adopting the following recurrent constraint for the relaxation times: $\lambda_{\mathrm{i}+1}=10 \lambda_{\mathrm{i}}$.

\section{Results and discussion}

\subsection{Simple systems}

The first section regards the rheological characterization of aqueous simple systems, containing only scleroglucan or Laponite at different concentrations and is aimed at drawing the necessary background for the analysis of mixed systems.

\subsubsection{Scleroglucan systems}

The shear thinning character of aqueous scleroglucan solutions increases with increasing polymer concentration $C$ and above $1 \mathrm{wt} \%$ the behavior becomes apparently plastic with a significant viscosity drop confined in a narrow stress interval, as typical for weak polymer gels. Fig. $1 \mathrm{~A}$ reports the experimental data in a log viscosity-log stress plot for polymer concentrations between 0.25 and $2 \mathrm{wt} \%$, together with the flow curves calculated with the RBC model. The increase in polymer concentration leads to the progressive shifting of the flow curves towards higher viscosity and stress values as well as a more marked viscosity drop in the shear thinning region. These effects can be quantitatively evaluated from the RBC parameters reported in Table 1 . The zero-shear rate viscosity $\eta_{0}$ and the critical stress $\sigma_{\mathrm{c}}$ increase with polymer concentration according to scaling laws. Their exponents are 5.6 and 2.88 , respectively. The abrupt increase of the $m$ value above $1 \%$ underlines the transition to apparently plastic behavior.

A satisfactory fitting quality is also obtained using the Cross equation for viscosity-shear rate correlation. The estimated $\eta_{0}$ values do not differ significantly from those given by the RBC model (see Table 1). The values of the critical shear rate $\dot{\gamma}_{c}$ (equal to the reciprocal of the characteristic time $\lambda$ ) and of the power law exponent $n$ clearly indicate how much the polymer concentration affects the onset and the degree of shear thinning, respectively. 
A)

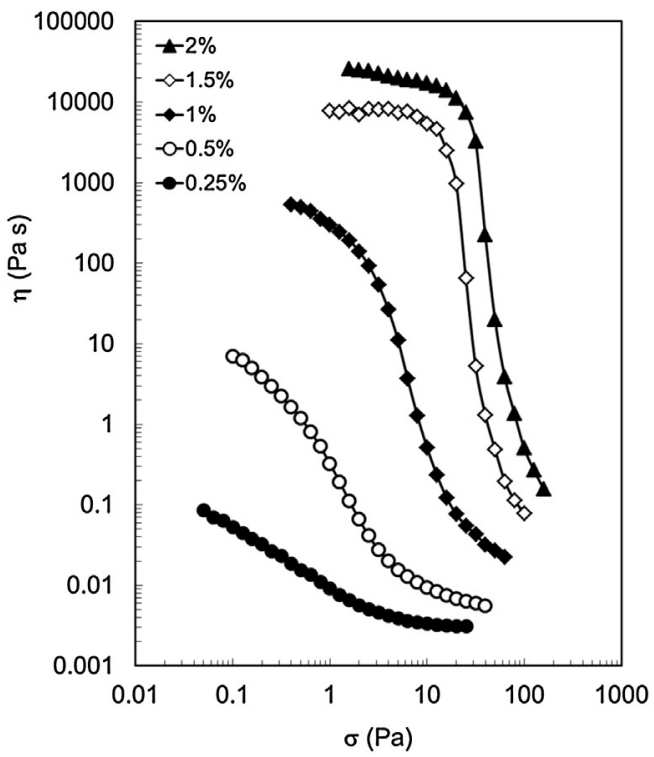

B)

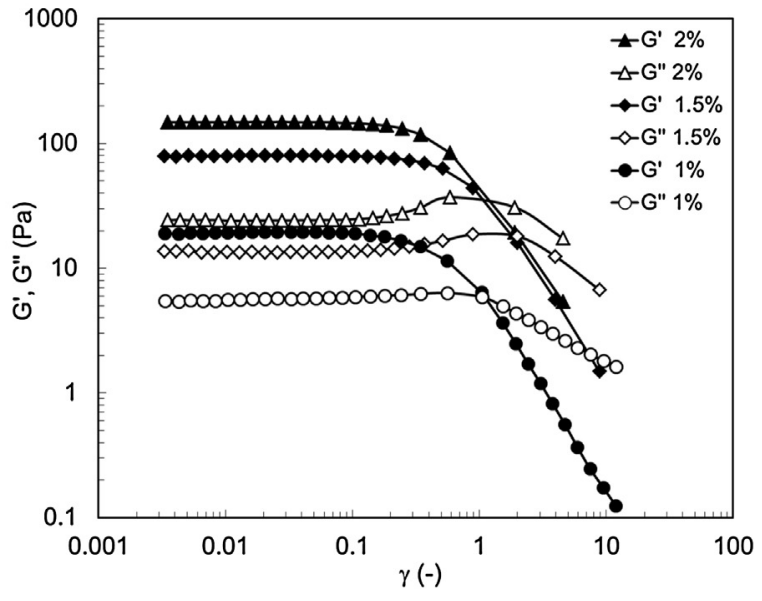

C)

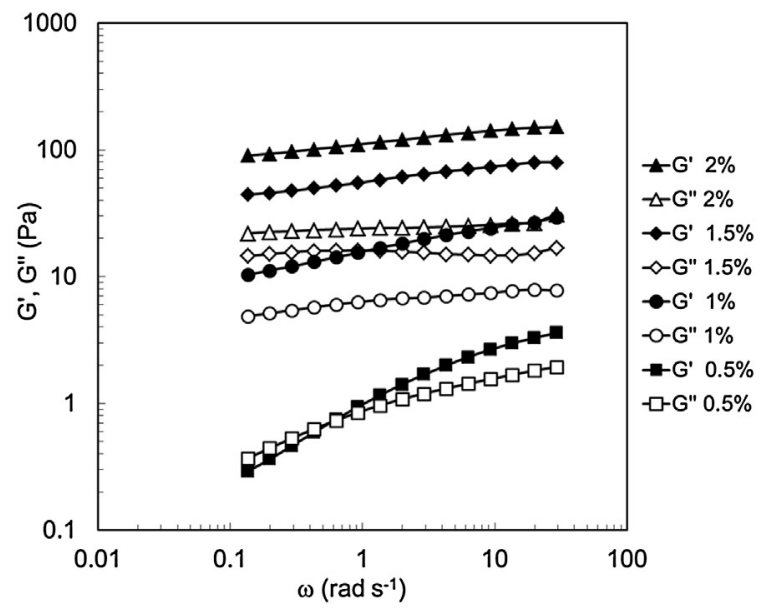

Fig. 1. A) Flow curves of scleroglucan systems at different polymer concentrations (from 0.25 to $2 \mathrm{wt} \%$ ); B) Strain dependence of the viscoelastic moduli for three scleroglucan systems (1, 1.5 and $2 \mathrm{wt} \%)$; C) Mechanical spectra of scleroglucan systems at different polymer concentrations $(0.5,1,1.5$ and $2 \mathrm{wt} \%)$.

The sol-gel transition induced by increasing polymer concentration can be more properly individuated by examining the changes in the viscoelastic responses obtained from both stress and frequency sweeps. Fig. 1B illustrates the strain dependence of the viscoelastic moduli for three different concentrations. Above $1 \%$ deformation, the border of the linear viscoelastic regime is marked by an initial increase of the loss modulus $G^{\prime \prime}$ followed by a subsequent decrease in strongly nonlinear conditions. This LAOS (Large Amplitude Oscillatory Shear) behavior characterized by a weak strain overshoot has been classified as type III (Hyun, Kim, Ahn, \& Lee, 2002) and is typical of weak gels, such as xanthan, and several other weakly structured fluids as well.

Even more evident are the changes of the mechanical spectra as polymer concentration increases (see Fig. 1C). Below $1 \mathrm{wt} \%$ the linear viscoelastic response is typical of ordinary polymer solutions with a crossover condition detectable within the experimental window. Conversely, at scleroglucan concentrations higher than $1 \mathrm{wt} \%$ it resembles those of other weak polymeric gels (Lapasin, 2015; Lapasin \& Pricl, 1995): $G^{\prime}$ exceeds $G^{\prime \prime}$ over the whole $\omega$ range explored, and the profiles of $G^{\prime}(\omega)$ and $G^{\prime \prime}(\omega)$ are nearly parallel with a slight frequency dependence. Using the generalized Maxwell model for data fitting, these concentration effects result in a progressive transition of the relaxation time spectrum from a wedge-type towards a box-type distribution owing the increasing weight of longer relaxation times.

\subsubsection{Laponite systems}

An increase in nanoclay concentration gives origin to a sharp transition in both the flow properties and the linear viscoelastic behavior of aqueous Laponite systems. Below $0.75 \%$ dilute dispersions are almost Newtonian or slightly shear thinning and their viscoelastic properties are practically undetectable with conventional instruments and methods. At higher concentrations, the shear behavior is decisively plastic since a dramatic viscosity drop (of several decades) is confined within a very narrow stress interval (see Fig. 2A), and the mechanical spectrum is typical of gels or other arrested states, with an evident prevalence of $G^{\prime}$ over $G^{\prime \prime}$ (more than one order of magnitude) along the whole frequency window (see Fig. 2B). As expected, stress sweep tests showed that concentrated Laponite dispersions (above $0.75 \%$ ) exhibit a LAOS behavior of type III.

The concentration effects on the rheological properties of scleroglucan systems are more gradual and unaffected by storage time, whereas the sharp sol-gel transition of Laponite systems depends on time elapsed since sample preparation and progressively shifts to lower clay concentration threshold with increasing aging time. Previous considerations are referred to experimental data determined on aged systems ( 9 days after preparation).

Fig. 3A shows how the flow curve of the $2 \%$ Laponite dispersion gradually changes its shape with increasing aging time. After few days the behavior becomes plastic and the transition from the low shear Newtonian plateau to the shear thinning region shifts along both axes towards higher viscosities and stresses. These changes can be conveniently measured through the progressive and parallel increase of the two RBC parameters, $\eta_{0}$ and $\sigma_{c}$, as illustrated in Fig. 3B. The change from shear thinning to plastic behavior is even better recognizable from the time evolution of the parameter $m$, which reaches high vales after few days.

As discussed previously, time elapsed after preparation plays an important role in the structural reorganization of Laponite nanoparticles, thus strengthening the gel behavior of concentrated dispersions or favoring the evolution toward a final arrested state at lower concentrations. Accordingly, aging time affects also the solgel transition, shifting the threshold concentration towards lower values.

\subsection{Laponite-scleroglucan systems}

This section is dedicated to mixed systems, prepared according to different procedures and containing both scleroglucan and Laponite at sufficiently high concentrations to cause appreciable 
A)

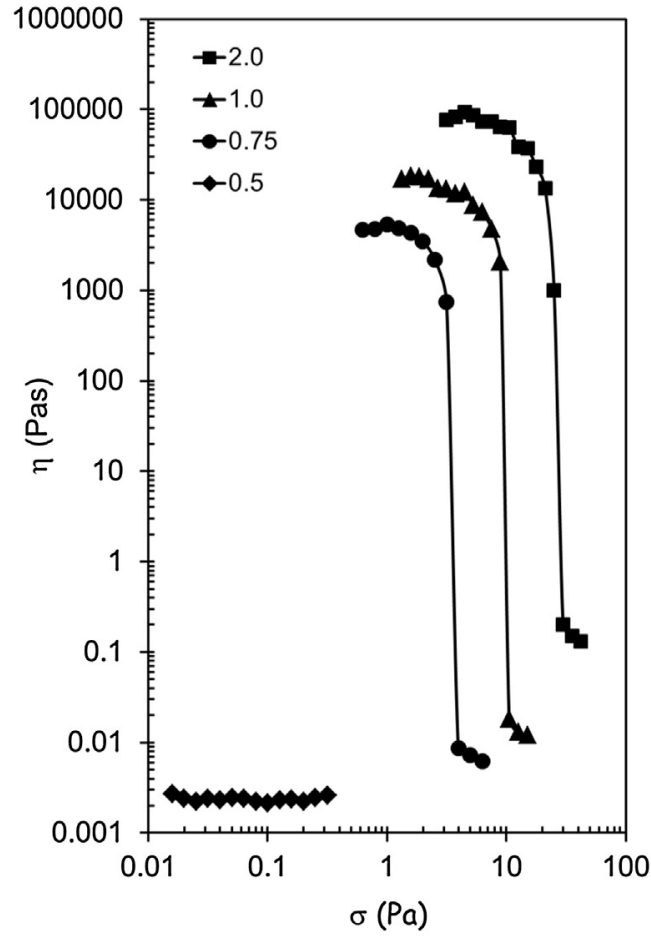

B)

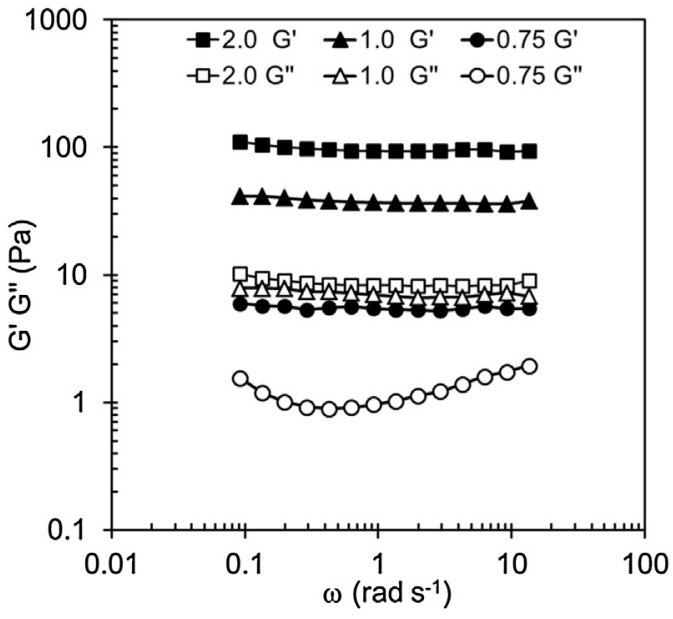

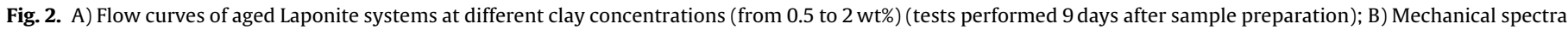
of aged Laponite systems at different clay concentrations (from 0.75 to $2 \mathrm{wt} \%$ ) (tests performed 9 days after sample preparation).
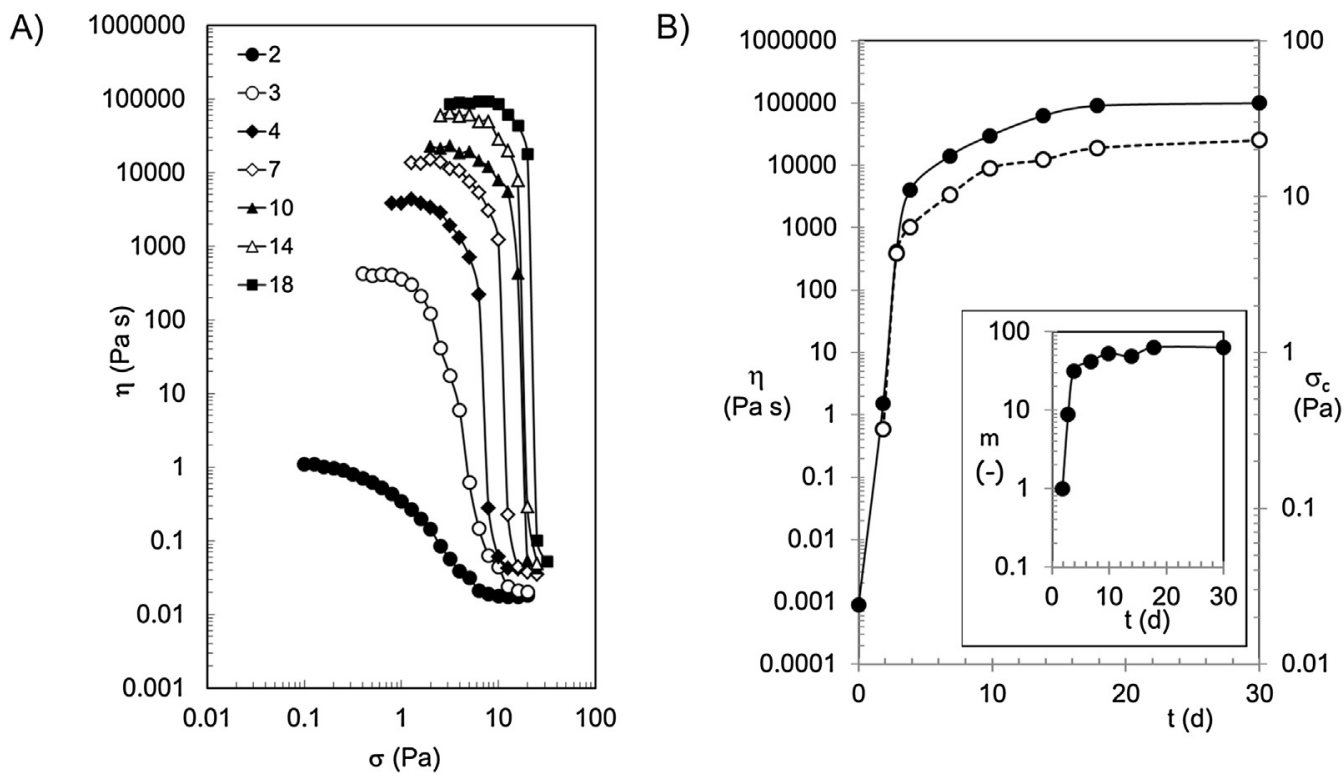

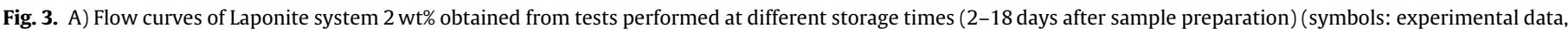

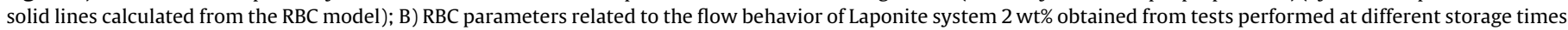
(2-18 days after sample preparation): zero-shear viscosity $\eta_{0}$ (filled circles), critical stress $\sigma_{\mathrm{c}}$ (open circles), parameter $m$.

rheological responses, immediately after their preparation or after enough elapsed time. The experimental tests have been aimed at examining how much the content and proportion of both components affect the viscoelastic and flow properties of the mixed system, also in view of practical applications in the biomedical field, where aqueous hydrogel matrices can be profitably used to formulate drug delivery systems in virtue of appropriate structural features and rheological behavior.

\subsubsection{Blends of fresh simple systems}

Scleroglucan-Laponite blends have been obtained by mixing fresh simple systems under mechanical agitation in different scleroglucan-Laponite proportions (3:1, 1:1, 1:3). The scleroglucan and Laponite systems have been prepared at the same polymer and clay weight percent concentration ( $2 \mathrm{wt} \%$ ), respectively, and blended two days after their preparation. Experimental tests have been repeatedly performed on mixed and simple systems during the following 16 days to examine the effects of aging time. 
A)

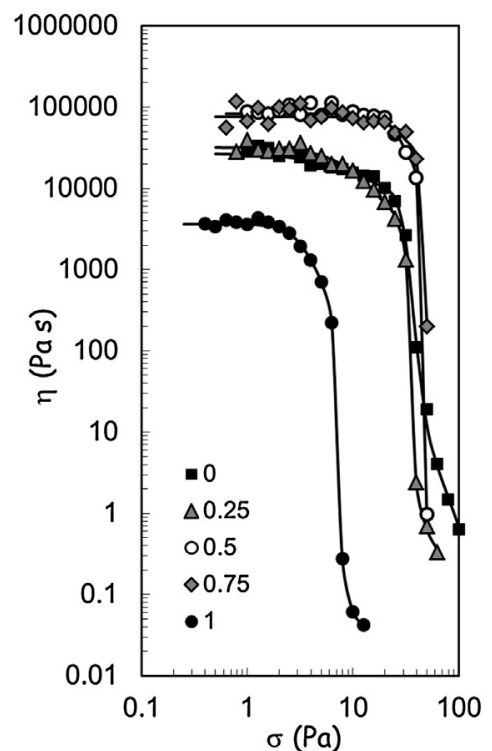

B)

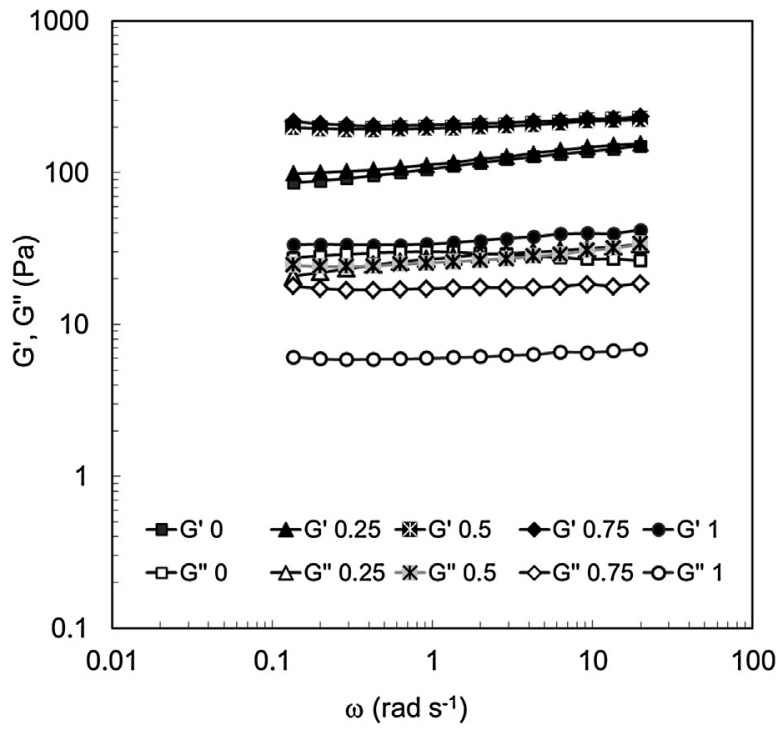

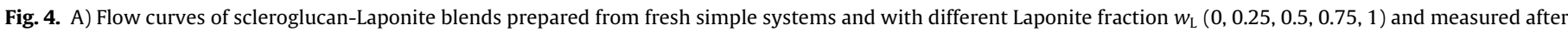

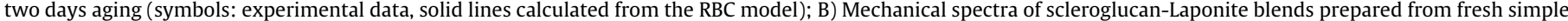

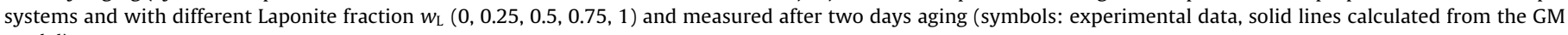
model).

Flow curves and mechanical spectra determined after two days of aging are compared in Fig. 4. All the systems exhibit a plastic behavior and then each of both RBC parameters, $\eta_{0}$ or $\sigma_{c}$, can be equivalently used to describe the blending effects on shear behavior owing to their interrelationship. Indeed, a change in mixing ratio leads to similar shifts of the flow curve along the two axes. After two days of aging the Laponite dispersion is characterized by the lowest values of both parameters among the examined systems, while the scleroglucan curve is placed at intermediate viscosity and stress levels. The maximum $\eta_{0}$ and $\sigma_{\mathrm{c}}$ (yield stress) values are attained for mixed systems (1:1 and $1: 3)$ with equal proportions of the two components or prevailing clay content in the mixture. Equivalent results are obtained using the Cross parameter $\eta_{0}$ whose values do not differ appreciably from those of the corresponding RBC parameter.

Thus, the comparison of the flow curves offers a first evidence of the synergistic effects of polymer-clay blending. Another proof can be obtained from linear viscoelastic behaviors. All the mechanical spectra are characterized by the predominance of the storage modulus and its slight or negligible frequency dependence (see Fig. 4B). The blending effects look quite similar to those evinced from flow behaviors. Indeed, both mixed systems $1: 1$ and 1:3 show an extended plateau with high $G^{\prime}$ values, one order of magnitude higher than $G^{\prime \prime}$. The GM model provides a quite satisfactory fit to data and its parameters are certainly suitable to analyze the changes associated with blending ratio. However, they can be replaced by the viscoelastic moduli, measured at a given frequency $(1 \mathrm{~Hz})$, which can serve the same purpose, even more conveniently, since the shape of the relaxation time spectrum does not change significantly with the blending ratio and only quantitative effects are observed.

The following Figures show how the viscous and viscoelastic parameters change with increasing aging time. Fig. 5A illustrates the dependence of the zero-shear rate viscosity on $w_{\mathrm{L}}$, which rep-
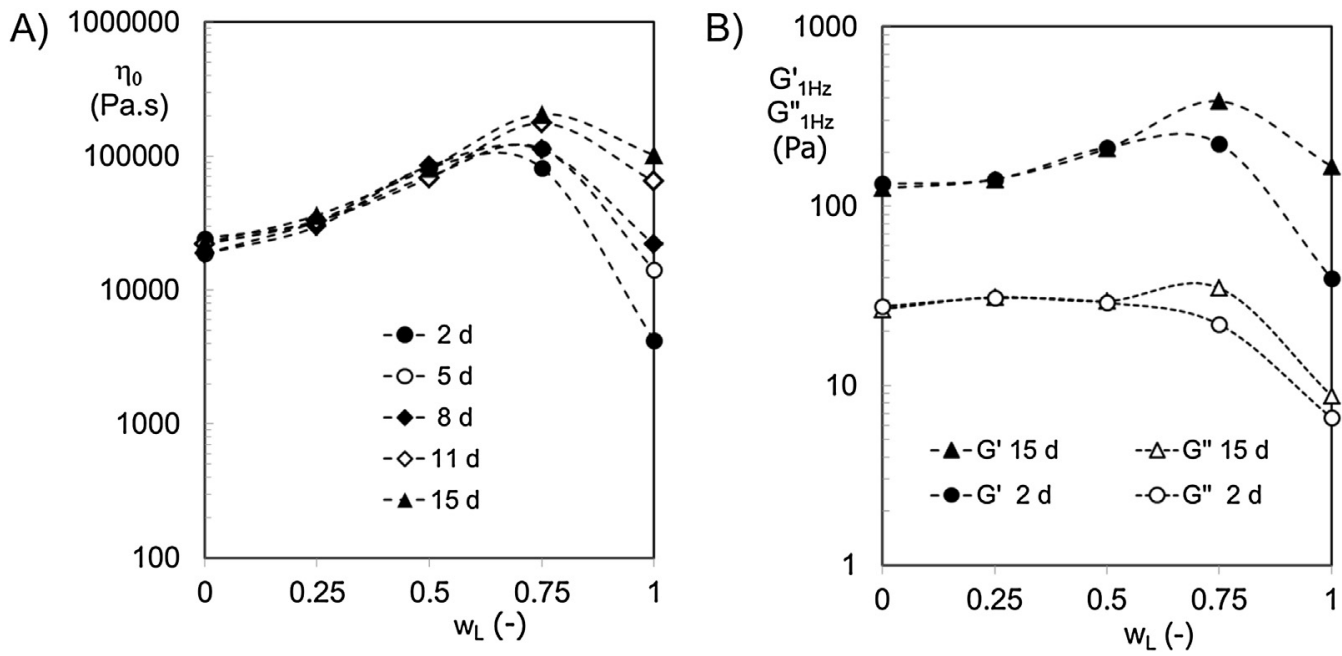

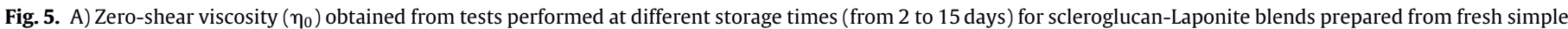

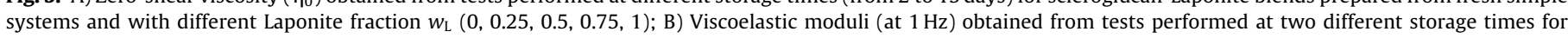
scleroglucan-Laponite blends prepared from fresh simple systems and with different Laponite fraction $w_{\mathrm{L}}(0,0.25,0.5,0.75,1)$ 

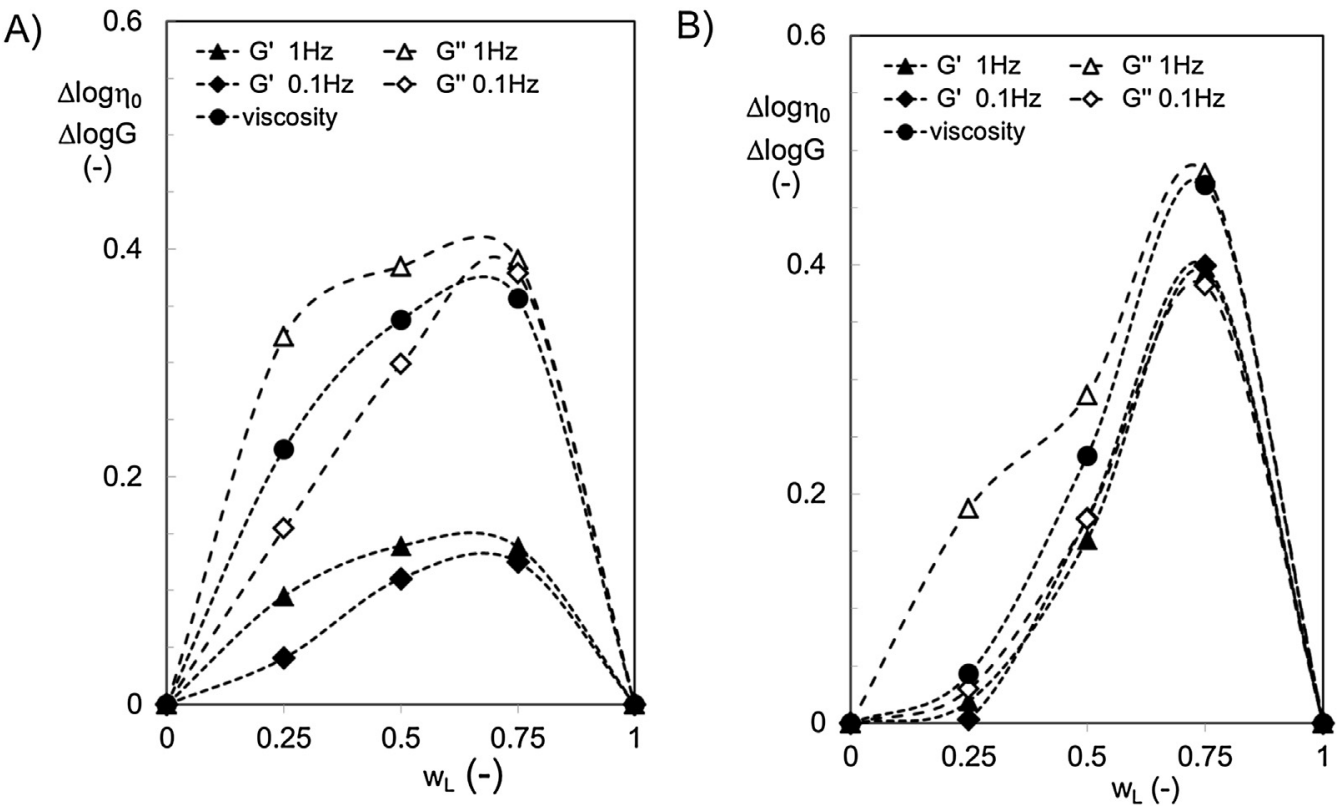

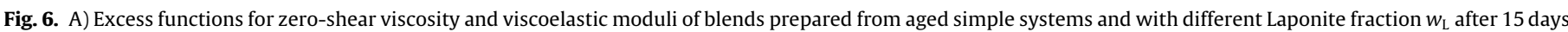

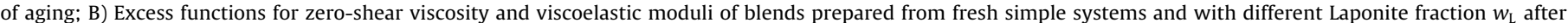
15 days of aging.

resents the weight fraction of simple Laponite dispersion present in the mixed system. The reported $\eta_{0}$ values have been calculated with the Cross equation from data obtained after different aging times $(2,5,8,11,15$ days). Fig. 5B regards the viscoelastic moduli $\left(G^{\prime}{ }_{1 \mathrm{~Hz}}, G^{\prime \prime}{ }_{1 \mathrm{~Hz}}\right)$ measured 2 and 15 days after preparation. The synergistic effects of blending can be clearly evinced by the profiles of $\eta_{0}, G^{\prime}{ }_{1 \mathrm{~Hz}}$ and, to a lesser extent, $G^{\prime \prime}{ }_{1 \mathrm{~Hz}}$, particularly two days after preparation. Increasing aging time leads to significant increments, in particular of $\eta_{0}$, for the simple Laponite system and even for the mixed system with prevailing Laponite content.

\subsubsection{Blends of aged simple systems}

Aging stability is decisively higher if blends are prepared from aged simple systems. The mixture parameters are similar to those exhibited by blends of fresh simple systems after 15 days of aging (Fig. 6). Here, the logarithms of $\eta_{0}, G^{\prime} 1 \mathrm{~Hz}$ and $G^{\prime \prime}{ }_{1 \mathrm{~Hz}}$ are chosen as the reference quantities for examining the blending effects. In analogy with the correlation equations, often used for dynamic or kinematic viscosities of Newtonian liquids mixtures (Grunberg \& Nissan, 1949; McAllister, 1960), linear additivity is assumed for such quantities in absence of polymer-clay interactions and, consequently, positive or negative synergistic effects are measured by the excess functions. i.e. deviations from the linear mixing rule:

$\Delta Y=Y_{\exp }-Y_{i d} \quad\left(Y=\log \eta_{0}, \log G^{\prime}, \log G^{\prime \prime}\right)$

$Y_{i d}=Y_{S}+w_{L}\left(Y_{L}-Y_{S}\right)$

where the suffixes exp and id stand for measured value and value predicted by the linear mixing rule, respectively, and the values of the simple scleroglucan and Laponite systems $\left(Y_{S}\right.$ and $\left.Y_{L}\right)$ are linearly combined through the weight fraction $w_{\mathrm{L}}$ to calculate the ideal reference value.

It can be observed in Fig. 6 that blending effects on viscosity and moduli are similar, especially for blends prepared from fresh simple systems. $\log \eta_{0}, \log G^{\prime}$ and $\log G^{\prime \prime}$ deviations from the linear mixing rule show comparable profiles in dependence of blend composition. For both systems the effect of blending is maximal when blends are rich in Laponite and diminishes with decreasing nanoparticles content, in particular if $\eta_{0}$ e $G^{\prime \prime}$ are considered. Therefore, it may be concluded that the mode of preparation and storage conditions do affect blend rheological properties significantly, depending on the Laponite amount. Moreover, interesting conclusions may be drawn by observing viscosity ratios $\left(\eta_{0, R}\right)$, obtained by dividing $\eta_{0}$ values of blends by those of corresponding simple systems, which contain just one component (scleroglucan or Laponite) in the same concentration of the blend. In Fig. 7A the zero-shear viscosity profiles of blends prepared from aged simple systems are compared with those of aqueous Laponite dispersions and scleroglucan solutions having equal concentration of polymer or clay. In Fig. $7 \mathrm{~B}$ the viscosity ratios $\eta_{0, \mathrm{R}}$ of blends and relative viscosities $\eta_{0, r}$ of the corresponding simple systems are plotted vs both Laponite and scleroglucan concentrations. It can be noticed that the contribution of Laponite to the viscosity of scleroglucan matrices, measured by $\eta_{0, R}$, progressively increases with increasing Laponite amount and/or with decreasing polymer concentration in blend. At the same time the contribution of the polymer to the viscosity of the system gradually diminishes.

\subsubsection{Scleroglucan hydrogels added with Laponite nanoparticles}

The addition of Laponite to scleroglucan hydrogel ( $2 \mathrm{wt} \%$ ) produces only quantitative effects on its viscous and linear viscoelastic behaviors, which do not change qualitatively and remain similar to those of a typical physical polymer gel. As Laponite concentration increases, the shape of the mechanical spectra does not change significantly. Fitting data to the generalized Maxwell model shows that the equilibrium modulus $G_{\mathrm{e}}$ increases and also the contribution of the relaxation time spectra slightly increases, maintaining the same shape (box-type distribution). All the rheological parameters increase with increasing Laponite concentration. The effect tends to diminish progressively, when, despite their high concentration, nanoparticles, distributed within the polymer network, are no longer able to develop an extended aggregation structure, comparable to that of the corresponding simple Laponite system. The ratios of rheological parameters, obtained by dividing measured rheological parameters of blends by those of the simple scleroglucan system, which are shown in Fig. 8, support these observations. Quite similar increments are observed for the critical stress $\sigma_{\mathrm{c}}$ and the equilibrium modulus $G_{\mathrm{e}}$. 
A)

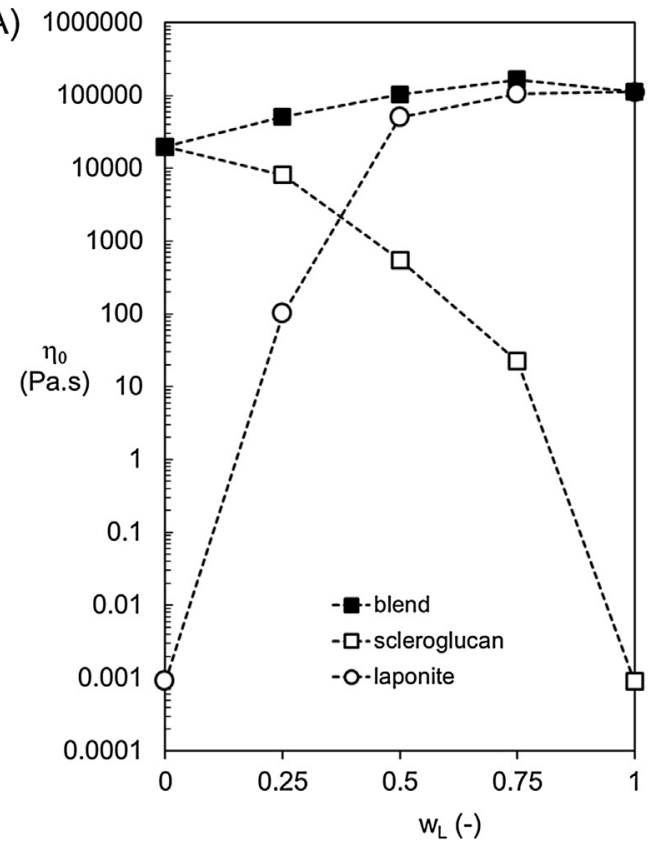

B)

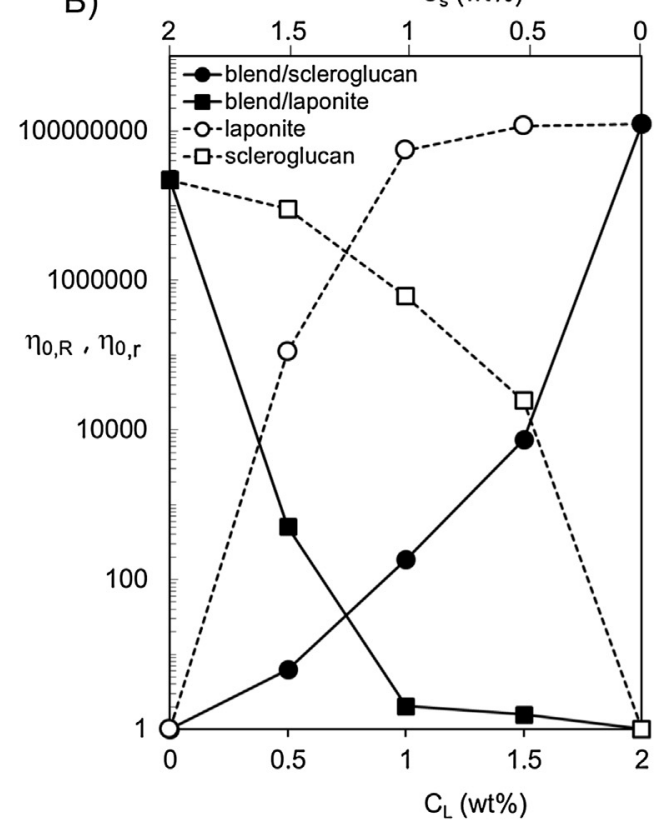

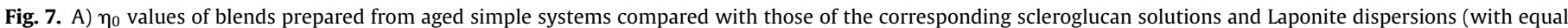

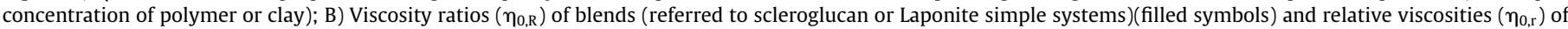
scleroglucan solutions and Laponite dispersions (open symbols) vs Laponite and scleroglucan concentrations $\left(C_{\mathrm{s}}\right.$ and $\left.C_{\mathrm{L}}\right)$.
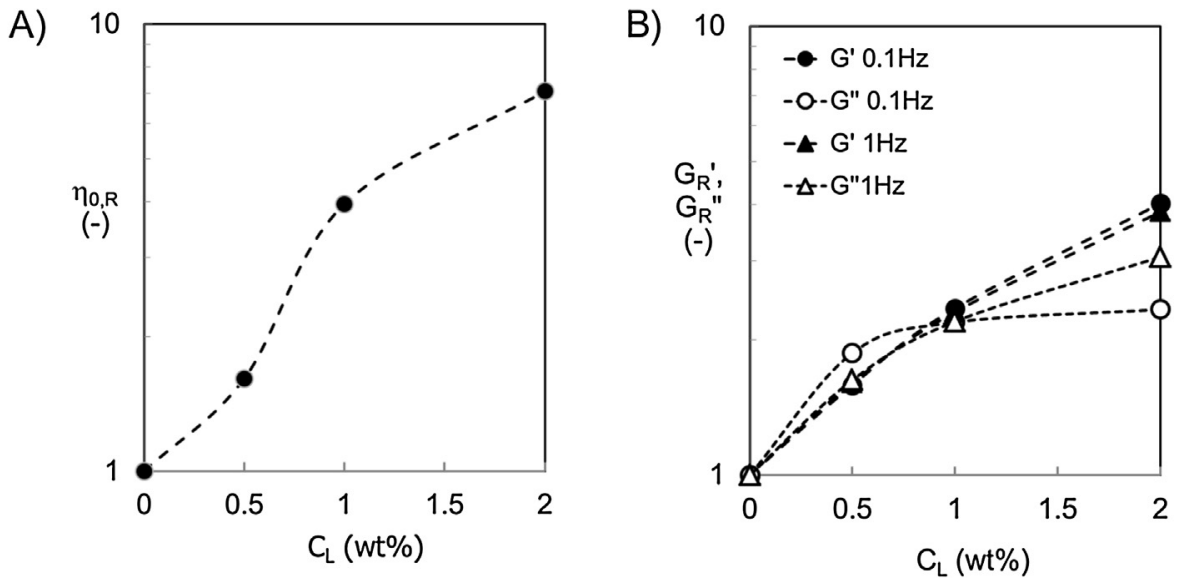

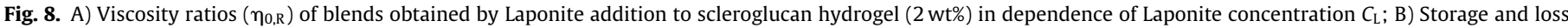
moduli ratios $\left(G_{\mathrm{R}^{\prime}}\right.$ and $\left.G_{\mathrm{R}^{\prime \prime}}\right)$ of blends obtained by Laponite addition to scleroglucan hydrogel ( 2 wt\%) in dependence of Laponite concentration $C_{\mathrm{L}}$.

As it can be noted, the increase of viscosity is significant and greater than those of the corresponding moduli parameters. Indeed, for the maximal Laponite addition ( $2 \mathrm{wt} \%$ ) the zero shear viscosity is seven times higher than that of the polymeric matrix, while it is $70 \%$ higher than that of the aqueous Laponite dispersion at $2 \mathrm{wt} \%$. However, it should be also underlined that the $\eta_{0, R}$, value of the mixed system, obtained by Laponite addition to the scleroglucan hydrogel matrix, is significantly lower (for about 8 orders of magnitude) than the $\eta_{0, r}$ value of the Laponite dispersion (see previous figure). The rheological responses of the hydrogel with $2 \mathrm{wt} \%$ Laponite added are much close to those exhibited by blends of fresh or aged simple systems with 1:3 scleroglucan-Laponite proportion in spite of their lower contents of both components.

It may be assumed, that by adding Laponite to a previously formed scleroglucan hydrogel, the individual clay nanoparticles, although capable of aggregation, are distributed within the meshes of triplex network, thus contributing to the rheological response of the system not differently from other particulate fillers.

\section{Conclusions}

The study carried out has shown that the blending of mature Laponite dispersions with weak gels of scleroglucan can give hybrid systems of rheological properties superior to those predictable by applying the linear mixing rule for corresponding simple systems. The synergistic effects previously explained in the introduction are particularly evident for Laponite rich blends. For example, for blends with Laponite/scleroglucan weight ratio 3:1 the measured rheological parameters (zero shear viscosity and viscoelastic moduli) were from two to three times higher than those predicted by the mixing rule.

Different types and grades of hydrogel structures are obtained by varying the ratio of the two components. For investigated blends the progressive increase of the content of Laponite particles corresponds to an equal reduction of the scleroglucan amount. The increase of the Laponite amount in a blend, beyond a critical threshold concentration, implies an increasing number and size 
of nanoparticle aggregates whose rheological contribution can not only compensate but also overcome the negative effects due to decreasing polymer contribution.

On the other hand, by adding Laponite nanoparticles into a gel matrix of scleroglucan ( $2 \mathrm{wt} \%$ ) significant increases in viscosity and viscoelastic moduli are obtained. However, these incremental effects diminish with increasing nanoclay addition.

The rheological responses of such mixed system having a Laponite content of $2 \mathrm{wt} \%$ are only slightly higher than those of the corresponding simple dispersion of Laponite ( $2 \mathrm{wt} \%$ ) in water. They are also very similar to those of blends obtained from simple systems of Laponite and scleroglucan with blending ratio 3:1 (overall amount of Laponite and scleroglucan represents $2 \mathrm{wt} \%$, fresh or matured blends), which contain less Laponite and scleroglucan.

Therefore, it may be concluded that hydrogels of comparable rheological behavior can be prepared by blending simple Laponite and scleroglucan aqueous systems, in different ratios and by different modes of preparation, or by adding Laponite powder into a gel matrix of scleroglucan. These observations open up interesting prospects of using hydrogels based on Laponite and scleroglucan in the biomedical field, in particular for the preparation of controlled release systems, and hint at possible improvements with respect to performance of scleroglucan hydrogels. Despite the comparable viscous and viscoelastic properties of such gel systems, it is reasonable to assume that their structural characteristics, if examined on different length scales, are substantially dissimilar. Consequently, also the diffusivity of an active substance within the complex gel structure with different degree of heterogeneity and interpenetration of structures of Laponite and scleroglucan should be significantly affected. Forthcoming structural investigations, using low-field NMR tests and studies on the active drug release, will allow us to better assess the opportunities for use of such hybrid systems in the field of drug delivery and, possibly, to optimize the formulation of the Laponite/scleruglucan hydrogel.

\section{Acknowledgements}

The authors acknowledge the financial support from the Slovenian Research Agency (research core funding No. P2-0191).

\section{References}

Baba Hamed, S., \& Belhadri, M. (2009). Rheological properties of biopolymers drilling fluids. Journal of Petroleum Science and Engineering, 67, 84-90.

Baghdadi, H. A., Sardinha, H., \& Bhatia, S. R. (2005). Rheology and gelation kinetics in Laponite dispersions containing poly(ethylene oxide). Journal of Polymer Science Part B: Polymer Physics, 43, 233-240.

Baghdadi, H. A., Jensen, E. C., Easwar, N., \& Bhatia, S. R. (2008). Evidence for re-entrant behavior in Laponite-PEO systems. Rheologica Acta, 47, 121-127.

Bais, D., Trevisan, A., Lapasin, R., Partal, P., \& Gallegos, C. (2005). Rheological characterization of polysaccharide-surfactant matrices for cosmetic $\mathrm{O} / \mathrm{W}$ emulsions. Journal of Colloid and Interface Science, 290, 546-556.

Bluhm, T. L., Deslandes, Y., Marchessault, R. H., Pérez, S., \& Rinaudo, M. (1982). Solid-state and solution conformation of scleroglucan. Carbohydrate Research, $100,117-130$.

Bohn, J. A., \& BeMiller, J. N. (1995). (1 $\rightarrow 3)-\beta-D-G l u c a n s$ as biological response modifiers: A review of structure-functional activity relationships. Carbohydrate Polymers, 28, 3-14.

Coviello, T., Grassi, M., Lapasin, R., Marino, A., \& Alhaique, F. (2003). Scleroglucan/borax: Characterization of a novel hydrogel system suitable for drug delivery. Biomaterials, 24, 2789-2798.

Coviello, T., Palleschi, A., Grassi, M., Matricardi, P., Bocchinfuso, G., \& Alhaique, F. (2005). Scleroglucan: A versatile polysaccharide for modified drug delivery. Molecules, 10, 6-33.

Cross, M. M. (1965). Rheology of non-Newtonian fluids: A new flow equation for pseudoplastic systems. Journal of Colloid Science, 20, 417-423.

Cummins, H. Z. (2007). Liquid, glass, gel: The phases of colloidal Laponite. Journal of Non-Crystalline Solids, 353, 3891-3905.

Daga, V. K., \& Wagner, N. J. (2006). Linear viscoelastic master curves of neat and Laponite-filled poly(ethylene oxide)-water solutions. Rheologica Acta, 45, 813-824
Davila, J. L., \& d'Ávila, M. A. (2017). Laponite as a rheology modifier of alginate solutions: Physical gelation and aging evolution. Carbohydrate Polymers, 157, $1-8$.

de Bruyn, J. R., Pignon, F., Tsabet, E., \& Magnin, A. (2008). Micron-scale origin of the shear-induced structure in Laponite-poly(ethyleneoxide) dispersions. Rheologica Acta, 47, 63-73.

De Lisi, R., Gradzielski, M., Lazzara, G., Milioto, S., Muratore, N., \& Prévost, S. (2008). Aqueous Laponite clay dispersions in the presence of poly(ethylene oxide) or poly(propylene oxide) oligomers and their triblock copolymers. The Journal of Physical Chemistry B, 112, 9328-9336.

Ellis, S. B. (1927). Thesis. USA: Lafayette College, Pa. Cited in: Matsuhisa, S., \& Bird R. B. (1965). Analytical and numerical solutions for laminar flow of the Non-Newtonian Ellis fluid. American Institute of Chemical Engineers Journal, 11, 588-595.

Fariña, J. I., Siñeriz, F., Molina, O. E., \& Perotti, N. I. (2001). Isolation and physicochemical characterization of soluble scleroglucan from Sclerotium rolfsii. Rheological properties, molecular weight and conformational characteristics. Carbohydrate Polymers, 44, 41-50.

Fitch, F. R., Jenness, P. K., \& Rangus, S. E. (1991). Rheological study of blends of Laponite and polymer thickeners. In R. A. F. Williams, \& N. C. de Jaeger (Eds.) Advances in measurement and control of colloidal processes (pp. 292-310). Oxford: Butterworth-Heinemann.

Gabriel, J.-C. P., Sanchez, C., \& Davidson, P. (1996). Observation of nematic liquid-crystal textures in aqueous gels of smectite clays. The Journal of Physical Chemistry, 100, 11139-11143.

Gallino, G., Guarneri, A., Poli, G., \& Xiao, L. (1996). Scleroglucan biopolymer enhances WBM performances. In SPE annual technical conference and exhibition (pp. 105-119). http://dx.doi.org/10.2118/36426-ms

Grassi, M., Lapasin, R., Coviello, T., Matricardi, P., Di Meo, C., \& Alhaique, F. (2009) Scleroglucan/borax/drug hydrogels: Structure characterisation by means of rheological and diffusion experiments. Carbohydrate Polymers, 78, 377-383.

Grassi, M., Lapasin, R., \& Pricl, S. (1996). A study of the rheological behavior of scleroglucan weak gel systems. Carbohydrate Polymers, 29, 169-181.

Grassi, M., Lapasin, R., Pricl, S., \& Colombo, I. (1996). Apparent non-fickian release from a scleroglucan gel matrix. Chemical Engineering Communications, 155, 89-112.

Grunberg, L., \& Nissan, A. H. (1949). Mixture law for viscosity. Nature, 164, 799-800.

Hyun, K., Kim, S. H., Ahn, K. H., \& Lee, S. J. (2002). Large amplitude oscillatory shear as a way to classify the complex fluids. Journal of Non-Newtonian Fluid Mechanics, 107, 51-65.

Jabbari-Farouji, S., Tanaka, H., Wegdam, G. H., \& Bonn, D. (2008). Multiple nonergodic disordered states in Laponite suspensions: A phase diagram. Physical Review E, 78, 061405

Kishore, S., Chen, Y., Ravindra, P., \& Bhatia, S. R. (2015). The effect of particle-scale dynamics on the macroscopic properties of disk-shaped colloid-polymer systems. Colloids and Surfaces A: Physicochemical and Engineering Aspects, 482, 585-595.

Kulawardana, E. U., Koh, H., Kim, D. H., Liyanage, P. J., Upamali, K., Huh, C., et al. (2012). Rheology and transport of improved EOR polymers under harsh reservoir conditions. In SPE improved oil recovery symposium http://dx.doi.org/ 10.2118/154294-ms

Lapasin, R., \& Maiutto, A. (2006). Rheology of aqueous Laponite-polymer mixtures. In AERC 2006: 3rd annual European rheology conference (p. 2006).

Lapasin, R., \& Pricl, S. (1995). Rheology of industrial polysaccharides: Theory and applications. London: Chapman \& Hall, London.

Lapasin, R., Pricl, S., \& Esposito, P. (1990). Rheological behaviour of scleroglucan aqueous systems in the solution and gel domains. In R. E. Carter (Ed.), Rheology of food, pharmaceutical and biological molecules (pp. 122-132). London and New York: Elsevier Applied Sciences.

Lapasin, R. (2015). Rheological characterization of hydrogels. In P. Matricardi, F. Alhaique, \& T. Coviello (Eds.), Polysaccharide hydrogels: Characterization and biomedical applications (pp. 83-137). Singapore: Pan Stanford Publishing.

Lapasin, R. (2016). Proprietà reologiche di dispersioni di Laponite in matrici acquose di xantano. Panta Rei, 14, 7-19.

Levitz, P., Lécolier, E., Mourchid, A., Delville, A., \& Lyonnard, S. (2000). Liquid-solid transition of Laponite suspensions at very low ionic strength: Long-range electrostatic stabilisation of anisotropic colloids. Europhysics Letters, 49, 672-677.

Lin-Gibson, S., Kim, H., Schmidt, G., Han, C. C., \& Hobbie, E. K. (2004). Shear-induced structure in polymer-clay nanocomposite solutions. Journal of Colloid and Interface Science, 274, 515-525.

Maiutto, A. (2005). Master Thesis. University of Trieste, Faculty of Engineering.

Malwitz, M. M., Butler, P. D., Porcar, L., Angelette, D. P., \& Schmidt, G. (2004). Orientation and relaxation of polymer-clay solutions studied by rheology and small-angle neutron scattering. Journal of Polymer Science B: Polymer Physics, 42, 3102-3112.

Matricardi, P., Onorati, I., Coviello, T., \& Alhaique, F. (2006). Drug delivery matrices based on scleroglucan-alginate-borax gels. International Journal of Pharmaceutics, 316, 21-28.

McAllister, R. A. (1960). The viscosity of liquid mixtures. American Institute of Chemical Engineers Journal, 6, 427-431.

Mongondry, P., Nicolai, T., \& Tassin, J. F. (2004). Influence of pyrophosphate or polyethylene oxide on the aggregation and gelation of aqueous Laponite dispersions. Journal of Colloid and Interface Science, 275, 191-196.

Mongondry, P., Tassin, J. F., \& Nicolai, T. (2005). Revised state diagram of Laponite dispersions. Journal of Colloid and Interface Science, 283, 397-405. 
Morariu, S., \& Bercea, M. (2015). Viscoelastic properties of Laponite RD dispersions containing PEO with different molecular weights. Revue Roumaine de Chimie, $60,777-785$.

Mourchid, A., Delville, A., Lambard, J., Lécolier, E., \& Levitz, P. (1995). Phase diagram of colloidal dispersions of anisotropic charged particles: Equilibrium properties, structure, and rheology of Laponite suspensions. Langmuir, 11, 1942-1950.

Mourchid, A., Lécolier, E., Van Damme, H., \& Levitz, P. (1998). On viscoelastic, birefringent, and swelling properties of laponite clay suspensions: Revisited phase diagram. Langmuir, 14, 4718-4723.

Palleschi, A., Bocchinfuso, G., Coviello, T. \& Alhaique, F. (2005). Molecular dynamics investigations of the polysaccharide scleroglucan: First study on the triple helix structure. Carbohydrate Research, 340, 2154-2162.

Pozzo, D., \& Walker, L. (2004). Reversible shear gelation of polymer-clay dispersions. Colloids and Surfaces A: Physicochemical and Engineering Aspects, 240, 187-198.

Rinaudo, M., \& Vincendon, M. (1982). 13C NMR structural investigation of scleroglucan. Carbohydrate Polymers, 2, 135-144.

Roberts, G. P., Barnes, H. A., \& Carew, P. (2001). Modelling the flow behaviour of very shear-thinning liquids. Chemical Engineering Science, 56, 5617-5623.

Ruzicka, B., \& Zaccarelli, E. (2011). A fresh look at the Laponite phase diagram. Soft Matter, 7, 1268-1286.

Ruzicka, B., Zulian, L., \& Ruocco, G. (2004). Routes to gelation in a clay suspension. Physical Review Letters, 93, 258301.

Ruzicka, B., Zulian, L., \& Ruocco, G. (2006). More on phase diagram of Laponite. Langmuir, 22, 1106-1111.

Ruzicka, B., Zaccarelli, E., Zulian, L., Angelini, R., Sztucki, M., Moussaid, A., et al. (2011). Observation of empty liquids and equilibrium gels in a colloidal clay. Nature Materials, 10, 56-60.

Savenko, V., Bulavin, L., Rawiso, M., Loginov, M., Vorobiev, E., \& Lebovka, N. I. (2013). Sedimentation stability and aging of aqueous dispersions of Laponite in the presence of cetyltrimethylammonium bromide. Physical Review E, 88, 052301.

Savenko, V., Bulavin, L., Rawiso, M., \& Lebovka, N. I. (2014). Aging of aqueous Laponite dispersions in the presence of sodium polystyrene sulfonate. Ukrainian Journal of Physics, 59, 589-595.

Schmidt, G., Nakatani, A. I., Butler, P. D., Karim, A., \& Han, C. C. (2000). Shear orientation of viscoelastic polymer-clay solutions probed by flow birefringence and SANS. Macromolecules, 33, 7219-7222.

Sveistrup, M., van Mastrigt, F., Norrman, J., Picchioni, F., \& Paso, K. (2016). Viability of biopolymers for enhanced oil recovery. Journal of Dispersion Science and Technology, 37, 1160-1169.

Tanaka, H., Meunier, J., \& Bonn, D. (2004). Nonergodic states of charged colloidal suspensions: Repulsive and attractive glasses and gels. Physical Review E, 69, 31404-31410.

Tanaka, H., Jabbari-Farouji, S., Meunier, J., \& Bonn, D. (2005). Kinetics of ergodic-to-nonergodic transitions in charged colloidal suspensions: Aging and gelation. Physical Review E, 71, 21402-21412.

Viñarta, S. C., François, N. J., Daraio, M. E., Figueroa, L. I. C., \& Fariña, J. I. (2007). Sclerotium rolfsii scleroglucan: The promising behavior of a natural polysaccharide as a drug delivery vehicle, suspension stabilizer and emulsifier. International Journal of Biological Macromolecules, 41, 314-323.

Yanaki, T., \& Norisuye, T. (1983). Triple helix and random coil of scleroglucan in dilute solutions. Polymer Journal, 15, 389-396.

Zebrowski, J., Prasad, V., Zhang, W., Walker, L. M., \& Weitz, D. A. (2003). Shake-gels: Shear-induced gelation of Laponite/PEO mixtures. Colloids and Surfaces A: Physicochemical and Engineering Aspects, 213, 189-197.

Zulian, L., Ruzicka, B., \& Ruocco, G. (2008). Influence of an adsorbing polymer on the aging dynamics of Laponite clay suspensions. Philosophical Magazine, 88, 4213-4221.

Zulian, L., Augusto De Melo Marques, F., Emilitri, E., Ruocco, G., \& Ruzicka, B. (2014) Dual aging behaviour in a clay-polymer dispersion. Soft Matter, 10, 4513-4521. 